\title{
Addendum to "Convergence of density and hybrid functional defect calculations for compound semiconductors"
}

\author{
Haowei Peng, David O. Scanlon, Vladan Stevanovic, Julien Vidal, Graeme W. Watson, and Stephan Lany \\ (Received 4 December 2017; published 13 December 2017)
}

DOI: 10.1103/PhysRevB.96.239903

This paper reported first-principles supercell calculations for the formation enthalpy $\Delta H$ of numerous charged defects in several semiconductors and insulators. The individual energy contributions leading to the final result for $\Delta H$, as given in Table IV of the original paper, were not included in the original submission. This Addendum provides detailed data allowing the interested reader to retrace the steps that lead to the final result, accessible through the Supplemental Material [1].

[1] See Supplemental Material at http://link.aps.org/supplemental/10.1103/PhysRevB.96.239903 for the atomic structures (primitive and supercells) and for a spreadsheet containing the individual energy contributions leading to the final results in $\Delta H$. 